\title{
EDITORIAL
}

\section{Non-valvular cardiovascular device infection}

About 3.25 million functioning pacemakers and 1,80,000 implantable cardioverter defibrillators have been implanted throughout the world as per registry. Despite of all precautions infection rates for these devices range from $1 \%$ to $7 \% .{ }^{1-4}$ With an increasing number of indications for use of permanent pacemakers (PPM), implantable cardioverterdefibrillators (ICD) and left ventricular assisted devices (LVADs) there has been an accelerated rate of device implantation. Management of cardiovascular device infection is a difficult challenge for both cardiologist and infectious diseases specialists. Reported incidence rates of cardiovascular device infection range from $0.13 \%$ to $19.9 \%$ and $0.0 \%$ to $0.8 \%$ for PPMs and ICDs, respectively. ${ }^{5-10}$ Infection may be primary in which the device and/or pocket itself is the source of the infection (usually due to the contamination at the time of implantation) or it may be secondary in which the leads are seeded due to bacteremia from a different source. Infection may be early when it develops within first month of implantation, or late when it occurs beyond first month of implantation and delayed when it develops beyond 12 months of implantation. Clinical presentations range from local signs at pocket site with pain, erythema, purulent drainage and wound dehiscence to blood-stream infection with high fever, sepsis and embolic/thrombotic events as well as indolent infection with low-grade fever and weight loss. Majority of patients present with pocket infection (52\%), 17\% with pocket infection and bacteremia and 23\% with device-related endocarditis. Risk factors for device infections are extended duration of implantation, recent manipulation such as generator exchange, old age, multiorgan failure, extended CCU staying, diabetes mellitus, malnutrition, malignancy, postoperative hematoma, prior treatment with anticoagulant and steroid as well as use of a temporary electrode. The most common causes of cardiovascular device infections are Coagulase-negative staphylococci (42\%) and Staphylococcus aureus (29\%), followed by gram-negative bacilli (9\%), including Klebsiella pneumoniae, Serratia marcescens, Pseudomonas aeruginosa, Stenotrophomonas maltophilia, Acinetobacter xylosoxidans, Acinetobacter baumanii, Citrobacter koseri, Morganella morganii, Hemophilus influenzae and Moraxella catarrhalis rarely fungus like Candida albicans and Aspergillus fumigatus infestations have been reported. In rare instances polymicrobial infections may present. ${ }^{11}$
Diagnosis is based on clinical symptoms as well as blood and generator pocket cultures. Transesophageal echo (TEE) recommended in all patients with positive blood cultures or before antibiotic therapy. Because transthoracic echo lacks sensitivity. Laboratory abnormalities include leukocytosis (WBC>10000), anemia, elevated ESR, and positive blood cultures. Complications include endocarditis, septic arthritis, osteomyelitis and pulmonary, brain, liver, perinephric and splenic abscesses.

Complete removal of cardiac device, if feasible, is recommended by the American Heart Association. ${ }^{5}$ Generator-tissue Gram stain and culture and lead tip culture should be obtained. The type of antimicrobial treatment depends on culture data. Early empiric therapy should cover nosocomial-acquired gram-positive organisms: vancomycin is a reasonable first choice, with modification based on culture data. Reassessment of need for cardiac device is recommended. Laser sheath technology allows photoablation of fibrous attachments without mechanical force and reduces the rate of complications associated with device removal. Most reports of successful eradication of infection without complete removal of the device occur with gram-negative bacteremia. ${ }^{12}$

Endocarditis demands device removal and pathogendirected parenteral therapy for 4-6 weeks. Duration of antibiotics should count from the date of device removal. Lead vegetation complicated by septic emboli/thrombosis, osteomyelitis, abscesses demands device removal and 4-6 weeks of antibiotics therapy. Bacteremia and negative TEE: demands device removal $\& 2$ weeks of antibiotics course (2-4 weeks of antibiotic treatment for S. aureus). ${ }^{13}$ Negative blood cultures without prior antibiotic therapy amounts the treatment for pocket infection with 10-14days of antibiotics. In most cases, removal of LVAD is not feasible. Long-term suppression is usually the goal until new device transplantation. After which, a course of antibiotic should use to cure the infection. If removal of cardiac device is not feasible, the addition of rifampicin may consider. Rifampicin may cause liver function abnormalities and may decrease anticoagulation effect of warfarin. If blood cultures remain persistently positive, treatment with antibiotics should last for at least 4 wks. If cardiac device removal is not possible, then long-term suppressive antibiotic therapy is recommended. 
Timing of reimplantation is based on clinical presentation and nature of pathogens present. Blood stream infection requires longer treatment. Reimplantation should done at a new, clean site. Adequate debridement and infection control demands prior to reimplantation. If endocarditis presented with valve vegetation, repeat blood cultures should done after device removal. Reimplantation may consider 14 days after negative blood culture. In case of lead vegetation, reimplantation may consider after negative blood cultures for 72 hours. Without any endocardial involvement, reimplantation may consider after 72 hours of negative blood cultures.

The new medical devices have greatly enhanced the care for patients with cardiovascular diseases. The use of these devices has not only increased the life span of patients, but also has improved quality of life. For some patients, the benefits of device availability are not fully realized because of complicating device infection. Moreover, these infections are often severe and, in some cases, life threatening. Cure of infection may be difficult in some cases to achieve if removal of the infected device is not a treatment option. Thus, future developments should be directed at designing nonvalvular, cardiovascular devices that are more resistant to infection and identifying antimicrobial agents that have enhanced activity in clearing infection from these indwelling medical devices. Methyl-silicone surface layers that are under development may minimize the accumulation of platelets, fibrinogen, and white blood cells; thereby reducing the mass of debris that is an excellent substrate for growth of microorganisms on prosthetic medical devices.

\section{Prof. Md. Abu Siddique}

Professor

Department of Cardiology

Bangabandhu Sheikh Mujib Medical University, Dhaka

\section{Dr. Mohammad Salman}

Assistant Professor

Anwar Khan Modern Medical College, Dhaka

\section{Reference}

1. Frame R, Brodman RF, Furman S, Andrews CA, Gross JN. Surgical removal of infected transvenous pacemaker leads. Pacing Clin Electrophysiol. 1993; 16:2343-48
2. Kearney RA, Eisen HJ, Wolf JE. Nonvalvular infections of the cardiovascular system, Ann Intern Med, 1994;121: 219-30

3. Lai KK, Fontecchio SA. Infections associated with implantable cardioverter defibrillators placed transvenously and via thoracotomies: epidemiology, infection control, and management. Clin Infect Dis. 1998; 27:265-69

4. Smith PN, Vidaillet HJ, Hayes JJ, Wethington PJ, Stahl L, Hull $\mathrm{M}$, et al. Infections with nonthoracotomy implantable cardioverter defibrillators: can these be prevented? Endotak Lead Clinical Investigators. Pacing Clin Electrophysiol. 1998; 21:42-55

5. Baddour LM, Bettmann MA, Bolger AF, et al. Nonvalvular cardiovascular device-related infections. Circulation 2003; 108:2015-31

6. Mela T, McGovern BA, Garan H, et al. Long-term infection rates associated with the pectoral versus abdominal approach to cardioverter-defibrillator implants. Am J Cardiol 2001; $88: 750-53$

7. Frame R, Brodman RF, Furman S, Andrews CA, Gross JN. Surgical removal of infected transvenous pacemaker leads. Pacing Clin Electrophysiol 1993; 16:2343- 48

8. Lai KK, Fontecchio SA. Infections associated with implantable cardioverter defibrillators placed transvenously and via thoracotomies: epidemiology, infection control, and management. Clin Infect Dis 1998; 27:265-69

9. Smith PN, Vidaillet HJ, Hayes JJ, et al. Infections with nonthoracotomy implantable cardioverter defibrillators: can these be prevented? Endotak Lead Clinical Investigators. Pacing Clin Electrophysiol 1998; 21:42-55

10. Hill PE. Complications of permanent transvenous cardiac pacing: a 14-year review of all transvenous pacemakers inserted at one community hospital. Pacing Clin Electrophysiol 1987; 10:564 -70

11. Chua JD, Wilkoff BL, Lee I, Juratli N, Longworth DL, Gordon SM. Diagnosis and management of infections involving implantable electrophysiologic cardiac devices. Ann Intern Med 2000;133:604-08

12. Lee JH, Geha AS, Rattehalli NM, et al. Salvage of infected ICDs: management without removal. Pacing Clin Electrophysiol 1996; 19:437-42

13. Wilson WR, Karchmer AW, Dajani AS, et al. Antibiotic treatment of adults with infective endocarditis due to streptococci, enterococci, staphylococci and HACEK microorganisms. American Heart Association. JAMA. 1995;274:1706-13 